\title{
MIMIMIZING THE COST OF USING TRACTORS AT AGRICULTURAL OPERATIONS
}

\author{
Rishat ZAYNAGABDINOV, Ildar GABITOV, Ilshat BAKIEV, Ildar GAFUROV, \\ Konstantin KOSTAREV
}

Department Automobiles and Machine-Tractor Complexes, Federal State Budgetary Educational Establishment of Higher Education “Bashkir State Agrarian University”, Ufa, Russia, zaynagabdinovri@ rambler.ru

\section{Abstract}

The paper shows the economic efficiency of the optimal use of technology in crop production. A totally new model has been developed ,that allows planning the operation of each tractor individually, as well as an integer programming method with Boolean variables based on the formation of a lexicographic sequence. The paper shows the calculation according to the optimization criterion of the minimum duration of the work. The developed method of integer programming is original because it allows to solve problems typical of agricultural planning. For this, the authors derived a formula for the formation of any member of the lexicographic sequence and developed the AgroMaster + computer program, which also carries out the search for the optimal solution using the method of truncated exhaustive search. Due to optimal planning, the total duration of agricultural operations is reduced by 1.7 times compared to their actual duration. The cost of additional products received per 1 moto-hour is 7.3 dollars. The testing the results of studies in one of the farms of the Republic of Bashkortostan showed the possibility of practical application of the above developments for optimal planning of tractors in any agricultural enterprises.

Keywords: crop production, DT-75 tractor, integer programming, T-402 tractor, optimal planning

\section{INTRODUCTION}

In the structure of the cost of agricultural products, the operating costs of the machine and tractor fleet reach $30-40 \%$. The optimal distribution of machinery for agricultural operations is one of the most important reserves to improve production efficiency. Due to the optimal planning, it is possible to minimize the cost of the work, reduce the duration of their implementation, and without investment. Solving of these problems in practice is very relevant given the scarcity of resources.

The indicators of the use of technology will vary significantly depending on the adopted objective function. For example, while minimizing the timing of agricultural activities, which is very important for a probable change in weather conditions, fuel consumption can be up to 1.5 times higher than with an optimal distribution according to the criterion of minimum fuel consumption [1-3]. The best plan from hundreds of possible ones can be chosen only through optimization mathematical calculations.

In literature, the problem of optimal planning of the use of technology is often reduced to the problem of a salesman [4, 5] Tractors and agricultural machinery as a resource are present in the tasks of the so-called maximin, where the optimization criterion is maximized or minimized depending on the purpose [6].
In [7] the search for an optimal plan for the use of technology is reduced to solving the problem of optimizing traffic flow. In most cases, the solution to the problem is non-integer, and the data obtained must be rounded. As a result, the optimality of the plan is violated, as well as the consistency of technologically related operations. A method for partial solution of this problem is proposed in the work, where a mixed integer programming algorithm is used [8]. However, this method can be used to solve particular problems of small dimension, and it is extremely difficult for practical use in the distribution of a large number of equipment.

When constructing models of optimal planning, many researchers lose sight of the need for additional time to transfer MTA from one field to another and to reaggregate. In [9] it is proposed to solve this problem by means of separate temporal and spatial planning.

Most of the proposed models initially assume that tractors and agricultural machines are located in one single point and are returned to this point every day at the end of work. However, in production, there may be cases when tractors are based at various points. In this case, it is necessary to introduce additional conditions into the model that take into account such a territorial spread of the bases. This moment is taken into account in [10]. 
In [11] the task of operational planning of the use of equipment is reduced to the problem of optimizing the composition of the machine-tractor fleet (MTF). Interesting methods for solving the problem in a network formulation are introduced in [12]. But such models are difficult to formalize.

To optimize the distribution of aggregates by type of work, a wide range of mathematical methods is used. This is due to differences in the description of production processes. The "simplex method" was most widely used [13, 14].

Modern researchers also propose modified linear programming algorithms that allow either to formally simplify [15] or to accelerate the solution of the problem [16].

In [17] research a model is proposed for selecting the best plan for using the technique for the case of multicriteria multimodal resource allocation based on a hybrid algorithm. However, in practice such a multichip statement of the problem is extremely rare.

As mentioned above, the authors neglect the requirement of integrality in solving practical problems, explaining that it is very difficult to solve problems of such large dimensions without taking this requirement into account. Therefore, if the task touches on the problem of integer variables, then a partially integer programming apparatus is used.

However, despite the variety of mathematical models for optimizing the MTA work plan, all of them are not sufficiently adapted for their practical use in the conditions of ordinary farms and have not received actual application [18]. The reason is as follows.

In real production, productivity and fuel consumption by tractors, even within the same brand, are not the same. This is due to the difference in the conditions of their work, the age of the equipment, qualifications and experience of machine operators. That is, even the same compositions of MTA have different performance characteristics (performance, fuel consumption). The analysis shows that the actual performance of MTA more than 2 times may differ from the normative. As a result, the plan drawn up can be not only non-optimal, but also unrealizable. Therefore, each tractor must be distributed by type of work separately - and this is an optimization problem with Boolean variables, for the solution of which the existing economic and mathematical models are not adapted (18). In addition, this in turn greatly increases the dimension of the problem, for the solution of which the existing mathematical apparatus is also not adapted. To solve discrete optimization problems, it is necessary to create special methods, such as cutting planes and branches and boundaries, but they are extremely difficult for software implementation.

The aim of the study is to reduce operating costs when performing agricultural mechanized work.
To achieve this goal, the following tasks were solved:

1. To develop a mathematical model for optimizing the distribution of MTA by type of work, allowing planning the work of each unit of equipment separately.

2. To establish dependencies for taking into account the influence of the "age" of tractors and other factors on the performance of the MTA.

3. To develop a mathematical apparatus for solving the optimization problem with Boolean variables in relation to the distribution of MTA by type of work.

\section{MATERIALS AND METHODS}

The development of the desired economic and mathematical model of the optimal MTA distribution by type of work is based on the above condition - it is necessary to plan the work of not tractor brands indicating their number, but each tractor individually. The mathematical part of the model was developed by the author and described in [19]. In a busy period of mechanized field work, when up to 15-20 different jobs are performed simultaneously, the most relevant criterion is the minimum total duration of operations. The objective function will look like this:

$$
\sum_{i=1}^{I} D_{i} \rightarrow \min
$$

The duration of the $i$ operation is:

$$
D_{i}=\frac{W_{i}}{w_{1 i} \cdot X_{1 i}+w_{2 i} \cdot X_{2 i}+w_{3 i} \cdot X_{3 i}+\ldots+w_{n i} \cdot X_{n i}}=\frac{W_{i}}{\sum_{n=1}^{N} w_{n i} \cdot X_{n i}}
$$

where $W_{i}$ is the volume of work on the $i$ operation, ha;

$w_{n i}$ is daily productivity of the unit with the tractor of the $n$th number (hereinafter the $n$th unit) in the $i$ operation, ha;

$X_{n i}$ is the required number of units in the $i$ operation, pcs.

$X_{n i}$ can take only two values - 0 or 1 .

The objective function (2) is linear fractional; to bring it to a linear dependence, we transform this expression by dividing the unit by it and denote:

$$
\frac{1}{D_{i}}=\frac{w_{1 i} \cdot X_{1 i}+w_{2 i} \cdot X_{2 i}+w_{3 i} \cdot X_{3 i}+\ldots+w_{n i} \cdot X_{n i}}{W_{i}}=d_{i}
$$

or in another way:

$\frac{1}{D_{i}}=\frac{W_{1 i}}{W_{i}} \cdot X_{1 i}+\frac{W_{2 i}}{W_{i}} \cdot X_{2 i}+\frac{W_{3 i}}{W_{i}} \cdot X_{3 i}+\ldots+\frac{W_{n i}}{W_{i}} \cdot X_{m i}=d_{i}$

Then the expression (1) will take the form: 


$$
\sum_{i=1}^{I} d_{i} \rightarrow \max
$$

The condition for using each tractor is written no more than once in the form:

$$
\begin{aligned}
& X_{11}+X_{12}+X_{13}+\ldots+X_{1 i}=\sum_{i=1}^{I} X_{1 i} \leq 1 \\
& X_{21}+X_{22}+X_{23}+\ldots+X_{2 i}=\sum_{i=1}^{I} X_{2 i} \leq 1 \\
& X_{31}+X_{32}+X_{33}+\ldots+X_{3 i}=\sum_{i=1}^{I} X_{3 i} \leq 1 \\
& X_{n 1}+X_{n 2}+X_{n 3}+\ldots+X_{n i}=\sum_{i=1}^{I} X_{n i} \leq 1
\end{aligned}
$$

The number of used agricultural machines and couplings should not exceed their number in the park:

$$
\begin{aligned}
& \sum_{i=1}^{I} \sum_{n=1}^{N} X_{n i} \cdot \lambda_{n i s} \leq N_{s} \\
& \sum_{i=1}^{I} \sum_{n=1}^{N} X_{n i \mu} \leq N_{\mu}
\end{aligned}
$$

where $N_{s}, N_{\mu}$ - respectively, the available number of agricultural machinery-implements of the $s$ th brand and coupling of the brand $\mu$;

$\lambda_{n i s}$ is the number of agricultural machines of the $s$ th type in the composition of $n$ units on the $i$ operation;

$X_{n i \mu}$ is the number of $n$ units on the $i$ th operation containing the $\mu$ brand coupling.

The condition for the coordination of the deadlines for technologically interconnected operations:

$$
D_{v_{1}} \leq D_{v_{2}} \leq \ldots \leq D_{v_{k-1}} \leq D_{v_{k}}
$$

where $v$ is the index of interrelated operations;

$k$ is No. of interconnected operations.

For example, pre-sowing cultivation, sowing and rolling crops are included in one block of interrelated operations. These operations are marked by two indices. The first index $v$ denotes the number of this block and is the same for these types of work (for example, $v=1$ ). The second $-k$ shows the order of work. In this case, for cultivation $k=1$, for sowing -2 , for rolling - 3 , that is, according to condition (9), the duration of the operation with a lower number $k$ should not exceed the duration of the operation with a higher number.

In this example, the condition must be met:

$$
D_{1_{1}} \leq D_{1_{2}} \leq D_{1_{3}}
$$

To enter a computer program, this expression should be represented as:

$$
\left\{\begin{array}{l}
d_{v_{1}}-d_{v_{2}} \geq 0 \\
d_{v_{2}}-d_{v_{3}} \geq 0 \\
\cdots \cdots \cdots \cdots \cdots \cdots \cdots \\
d_{v_{k-1}}-d_{v_{k}} \geq 0
\end{array}\right.
$$

The objective function, ensuring a minimum of total fuel consumption in operations, is written as:

$$
\sum_{i=1}^{I} Q_{i} \rightarrow \min
$$

where $Q_{i}$ is the fuel consumption at the $i$ th operation, $\mathrm{kg}$

Moreover

$$
Q_{i}=\left(w_{1 i} \cdot q_{1 i} \cdot X_{1 i}+w_{2 i} \cdot q_{2 i} \cdot X_{1 i}+w_{3 i} \cdot q_{3 i j} \cdot X_{3 i}+\ldots+w_{n i i} \cdot q_{i i} \cdot X_{n i j}\right) \cdot D_{i}
$$

where $q_{n i}$ is the rate of per hectare fuel consumption of the unit at the $i$ th operation, $\mathrm{kg} /$ ha.

Then the expression (12) takes the form:

$$
Q_{i}=\frac{w_{1 i} \cdot q_{1 i} \cdot X_{1 i}+w_{2 i} \cdot q_{2 i} \cdot X_{2 i}+w_{3 i} \cdot q_{3 i} \cdot X_{3 i}+\ldots+w_{n i} \cdot q_{n i n} \cdot X_{n i i}}{w_{1 i} \cdot X_{1 i}+w_{2 i} \cdot W_{2 i}+w_{3 i} \cdot X_{3 i}+\ldots+w_{n i} \cdot X_{n i}}
$$

In expanded form, function (12) is written as follows:

$$
\begin{aligned}
& \sum_{i=1}^{I} Q_{i}=\frac{w_{11} \cdot q_{11} \cdot X_{11}+w_{21} \cdot q_{21} \cdot X_{21}+w_{31} \cdot q_{31} \cdot X_{31}+\ldots+w_{n 1} \cdot q_{n 1} \cdot X_{n 1}}{w_{11} \cdot X_{11}+w_{21} \cdot X_{21}+w_{31} \cdot X_{31}+\ldots+w_{n 1} \cdot X_{n 1}}+ \\
& +\frac{w_{12} \cdot q_{12} \cdot X_{12}+w_{22} \cdot q_{22} \cdot X_{22}+w_{32} \cdot q_{32} \cdot X_{32}+\ldots+w_{n 2} \cdot q_{n 2} \cdot X_{21}}{w_{12} \cdot X_{12}+w_{22} \cdot X_{22}+w_{32} \cdot X_{32}+\ldots+w_{n 2} \cdot X_{n 2}}+ \\
& +\frac{w_{13} \cdot q_{13} \cdot X_{13}+w_{23} \cdot q_{23} \cdot X_{23}+w_{33} \cdot q_{33} \cdot X_{33}+\ldots+w_{n 3} \cdot q_{n 3} \cdot X_{n 3} \cdot w_{3}+\ldots}{w_{13} \cdot X_{13}+w_{23} \cdot X_{23}+w_{33} \cdot X_{33}+\ldots+w_{n 3} \cdot X_{n 3}}
\end{aligned}
$$

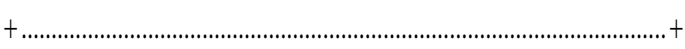

$$
\begin{aligned}
& +\frac{w_{1 i} \cdot q_{1 i} \cdot X_{1 i}+w_{2 i} \cdot q_{2 i} \cdot X_{2 i}+w_{3 i} \cdot q_{3 i} \cdot X_{3 i}+\ldots+w_{n i} \cdot q_{n i} \cdot X_{n i}}{w_{1 i} \cdot X_{1 i}+w_{2 i} \cdot X_{2 i}+w_{3 i} \cdot X_{3 i}+\ldots+w_{n i} \cdot X_{n i}} \rightarrow \min
\end{aligned}
$$

The objective function of the minimum of the reduced costs will have a similar form, but instead of the factors $w_{n i} \cdot q_{n i}$ in the expression (14) there will be the values of the reduced costs when the $n$th unit performs the $i$ operation.

The condition for the mandatory performance of the operation in implicit form can be written in the following form:

$$
d_{i}>0,
$$

To solve this problem, an algorithm for integer optimization with Boolean variables based on the construction of a lexicographic sequence (LS) has been developed. The latter is used in the Balash method [2], which underlies most integer programming applications.

LS is an ordered table of zeros and ones as a plan for using available resources. For example, the following LS shows how theoretically 3 units can be distributed in different jobs. 
Table 1. Lexicographic sequence for three variables

\begin{tabular}{|c|c|c|c|}
\hline \multirow{2}{*}{$\begin{array}{c}\text { Row } \\
\text { number }\end{array}$} & \multicolumn{3}{|c|}{ Unit designation and use plan } \\
\cline { 2 - 4 } & $\mathrm{X} 1$ & $\mathrm{X} 1$ & $\mathrm{X} 3$ \\
\hline 1 & 0 & 0 & 0 \\
\hline 2 & 1 & 0 & 0 \\
\hline 3 & 0 & 1 & 0 \\
\hline 4 & 1 & 1 & 0 \\
\hline 5 & 0 & 0 & 1 \\
\hline 6 & 1 & 0 & 1 \\
\hline 7 & 0 & 1 & 1 \\
\hline 8 & 1 & 1 & 1 \\
\hline
\end{tabular}

" 0 " in Table 1 means that the unit is not used, and " $1 "$ - that it is used. The total number of distribution options is calculated by the formula:

$$
N=2^{p}
$$

where $p$ is the number of distributed unit resources.

In our case, $p=3, N=2^{3}=8$

For each row, the target value is calculated, for example, costs. Next, the rows are sorted by increasing (decreasing) this target value and the optimal one is selected from them. Optimization criteria allow to exclude rows that are not suitable for consideration. For example, if $\mathrm{X} 1$ and $\mathrm{X} 2$ are units made up of the same tractor, then rows 4 and 8 are excluded from consideration, since the same tractor cannot be used simultaneously in two different jobs. Row number 1 with all zeros is also excluded, because it means that not a single unit is busy at work, that is, the work is not performed.

According to formula (17), the number of possible series grows in a power-law order, and the process of constructing an LS begins to become many times more complicated. For example, if $p=$ 10 , then $N=1024$. It is almost impossible to build such a row without getting confused. Through theoretical studies, a formula was derived for the formation of individual values of the lexicographic sequence, according to which, the value of a member of the row $a$ ( 0 or 1$)$ is equal to:

$$
a=\operatorname{int} \frac{n-1}{2^{k}-1}-\operatorname{int}\left[\frac{\text { int } \frac{n-1}{2^{k}-1}}{2}\right] \times 2
$$

where $n$ is the number of the row;

$k$ is the column number;

int is a function to select the integer part of a number.

According to this formula, the value of the variable $a$ is a function of the row number and column number. This allows you to significantly simplify the optimization program and reduce the time it takes to find a solution. For each operation, an LS block is compiled. Block formation allows to deliberately exclude from consideration those points that would necessarily be analyzed if using the Balash method. Then, for each row, the value of the objective function is calculated, according to which the rows are sorted in ascending order at minimization or in descending order at maximization. In the next step, combinations of compatible rows are selected. The rows may be incompatible if the restrictions are not met and if the conditions for using each tractor are violated only once. The rows enumeration program is designed so that the search for the optimum begins with the "best" options and moves to the "worst" ones until a satisfactory solution is obtained. All the mathematical part listed is put in the computer program "Agromaster +", developed by the author. The program allows to find the optimal solution using the method of truncated exhaustive search.

An important feature of the model and mathematical apparatus proposed above is the possibility of assigning the so-called "mandatory" tractors. "Mandatory" refers to specific tractors that the user, at his discretion, would like to assign to a particular operation or, on the contrary, would like the tractor operator not to work on this operation. This is especially important, since the final decision on the use of technology is made subjectively. The developed model allows subjective amendments to be laid in advance before solving the problem and to obtain the optimal plan being implemented.

With an increase in tractor life, the performance of the unit decreases. One reason for this is a decrease in the effective power of its engine. The studies of Professor Plaksin [20] found that this reduces losses in the transmission and due to slipping propulsors. Because of this, the actual traction efficiency of the tractor will be $2 \ldots 5 \%$ higher than in the calculated one.

Using these dependences, we derived the formula for adjusting the performance of the unit, taking into account the decrease in tractor engine power. To use it, you need to know how the value of the coefficient $K_{N e}$ from the operating time of the tractor changes. Taking to account to all amendments, the actual shift performance of the MTA will be equal to:

$\mathrm{W}$ rep $\mathrm{f}=\mathrm{W}$ rep $\mathrm{r} \cdot \mathrm{K} \mathrm{t} \cdot \mathrm{K} \mathrm{rug} \cdot \mathrm{K}$ rock $\cdot \mathrm{Kcc} \cdot \mathrm{Kh} \cdot \mathrm{K}$ trx

$$
\times(1,02 \ldots 1,05) \cdot\left(1-\frac{1-K_{N e}}{\mathrm{tn}}\right) \cdot \mathrm{K} \mathrm{td} \cdot \mathrm{Kr} \cdot \mathrm{r} \text { acm }
$$

where $W$ rep $r$ is the replaceable rate of production of MTA for normal working conditions, ha;

$\mathrm{K}$ t, $\mathrm{K}$ rug, $\mathrm{K}$ rock, $\mathrm{Kc} \mathrm{c}, \mathrm{K}$ h, $\mathrm{K}$ tr - correction factors to the production rate, taking into account, respectively, terrain, ruggedness by obstacles, rockiness, configuration complexity and field height above sea level, reduced traction on sandy soils (these coefficients are determined by known methods used to normalize mechanized work);

$\mathrm{K}$ td-coefficient taking into account the impact of tractor driver qualifications on the performance of the unit;

$\mathrm{K}$ r- coefficient of technical readiness of the 
tractor;

$\mathrm{r}$ acm- coefficient of operational reliability of an agricultural machine;

$n$ is the number of agricultural machines in the composition of the unit;

$\mathrm{t} n$ - traction efficiency of the tractor at rated engine power;

$$
K_{N e}=\frac{N e_{i}}{N e_{r}} \text { - coefficient taking into account the }
$$

decrease in the effective engine power as the service life of the tractor increases, where

$N e_{i}$-actual effective engine power of the tractor of the year of service, $\mathrm{kW}$;

$N e_{r}$ - rated engine power, $\mathrm{kW}$.

The factor $(1,02 \ldots 1,05) \cdot\left(1-\frac{1-K_{N e}}{\mathrm{t} \mathrm{n}}\right)$ in the formula (18) takes into account the effect of a decrease in the effective engine power with an increase in the tractor's "age" on the performance of the unit.

To obtain the values of individual parameters included in formula (18), experimental studies were conducted on the basis of agricultural enterprises of the Republic of Bashkortostan. The power efficiency of the engines of caterpillar tractors DT75 and T-402 was measured before and after the overhaul of the engines, as well as before and after their operational adjustment. For measurements, the IMDTS-M device was used.

To establish the dependence of tractor performance on the experience of tractor drivers, statistical data on the use of tractors in the farms of the Republic of Bashkortostan were analyzed, photo-timing observations were carried out. Preparation for observations and their implementation was carried out in accordance with the methodology of State Scientific Research Technical Institute.

\section{RESULTS}

The dependences presented in Fig. 1 are described by second-order polynomial functions. The analysis of the data shows that the power of the engines of the DT-75 brand tractors in the prerepair period (corresponding to 4000 ... 5000 motohours of operation) falls by $8 \ldots 10 \%, \mathrm{~T}-402$ - by $13 \%$. After repair, the engine power is not fully restored and is $3 \ldots 4 \%$ below the nominal value. The rate of power drop after repair is 1.54 times higher than before repair.

The engine power of the T-402 tractors decreases faster and in the pre-repair period (3500 ... 4100 moto-hours) drops by $13 \%$. After repair, power decreases 1.41 times faster than before repair.

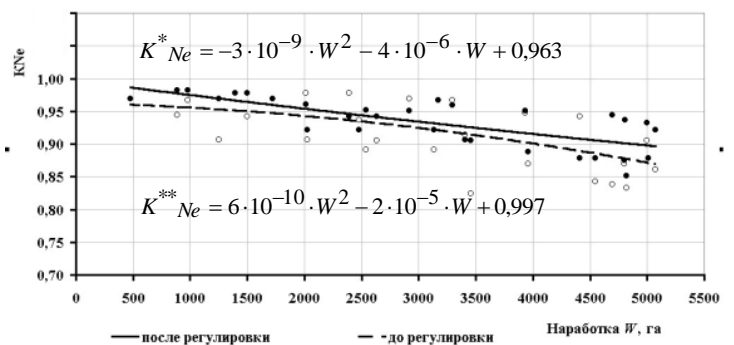

a)

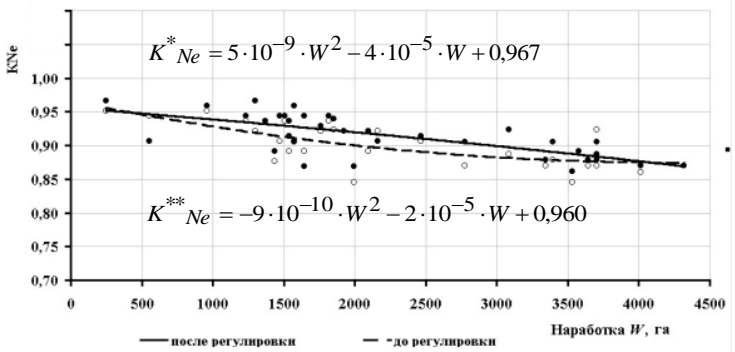

b)

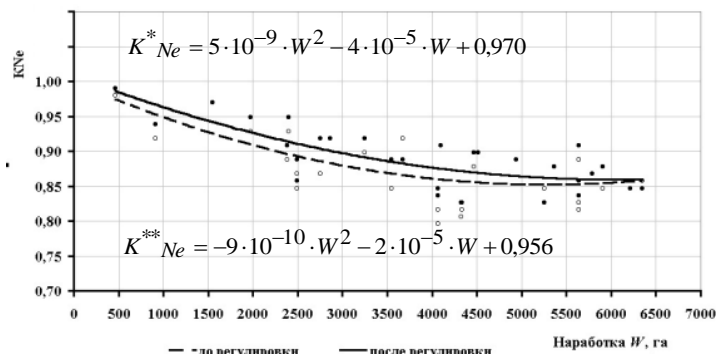

c)

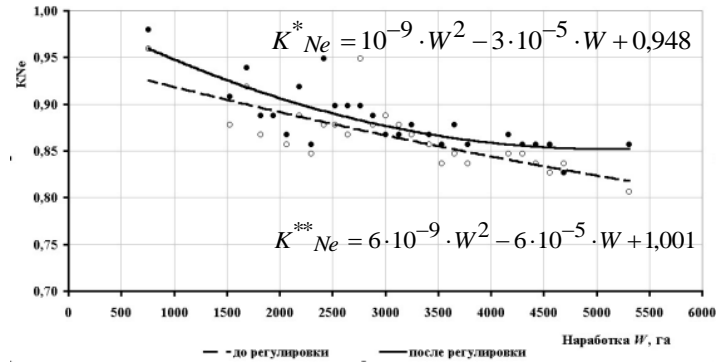

d)

Fig. 1. Dependence of the coefficient $K_{N e}$ value on the operating time of tractors: a) DT-75 before overhaul; b) DT-75 after overhaul; c) T-402 before overhaul; d) T-402 after overhaul; * - before adjustment; $* *$ - after adjustment.

Earlier studies found that among such indicators as age, work experience and tractor driver class, the work experience has a decisive influence on the degree of fulfillment of the production norm [19]. With this in mind, according to more than 100 observation sheets, the desired dependence has been identified (Fig. 2). 


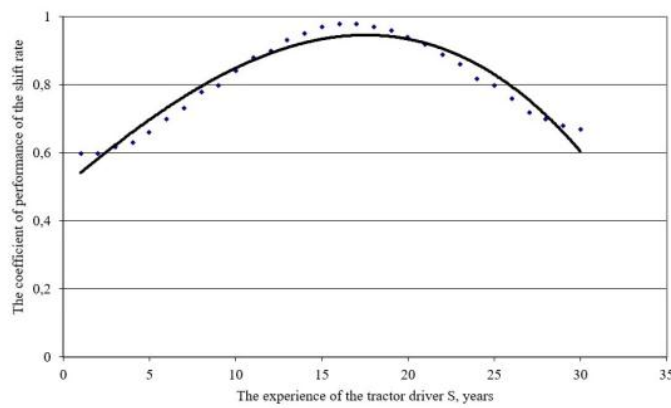

Fig. 2. The dependence of the coefficient of performance of the shift rate of development on the experience of the tractor driver

It is described by a third-order polynomial function:

$$
K W_{C M}=2 \times 10^{-5} S^{3}-0,0006 S^{2}+0,043 S+0,49
$$

the value of the multiple determination coefficient $\mathrm{R}^{2}$ is 0.9446 .

As can be seen from Fig. 2, in the first 1-3 years of work, the tractor operator on average fulfills the established production rate of $55-60 \%$. This is due to his inexperience. In addition, young tractor drivers are most often given old tractors for work. That is, two equally directed factors contribute to a decrease in the value of this coefficient. With an increase in the length of service, the degree of fulfillment of the production norm increases and reaches a maximum with an experience of 15-16 years. But its value still rarely reaches $100 \%$. This is explained by the fact that the norms are averaged for the entire economy and do not take into account the "age" of the equipment, the qualifications of the tractor driver and the specific characteristics of the field being cultivated. With an increase in the length of service, there is a decrease in the degree of fulfillment of the production norm. This is due to aging of the human body and rapid fatigue. Thus, the development of a 20-year-old tractor driver (experience 0-1 years) is approximately equal to the development of 50 years (experience 30 years).

According to the data on the operation of tractors in the farms of the republic, the dependence of the value of their coefficient of technical readiness of tractors on their service life was also investigated. It is known that the availability of equipment varies during the season, so it was studied in dynamics. The attempts to select equations to describe the desired dependencies using the classical technique of regression analysis, in particular the least squares method, were unsuccessful. In this regard, to solve the problem, neural network simulation was used. Note that previously, researchers did not use this relatively new type of modeling to solve such technical problems.

There are several main types of neural networks: multilayer perceptron (MLP); radial basis functions (RBF), linear networks (Linear), generalized regression networks (GRNN). The completeness of their description of the desired dependence is characterized by such an indicator as the performance of the network (Performance). Among these types of networks, a network search was organized to solve the problem in the STATISTICA Neural Network 4.0 software environment. As a result of the search, a network of RBF type was found with the highest quality equal to 0.98, which best describes the desired dependencies (Fig. 3).

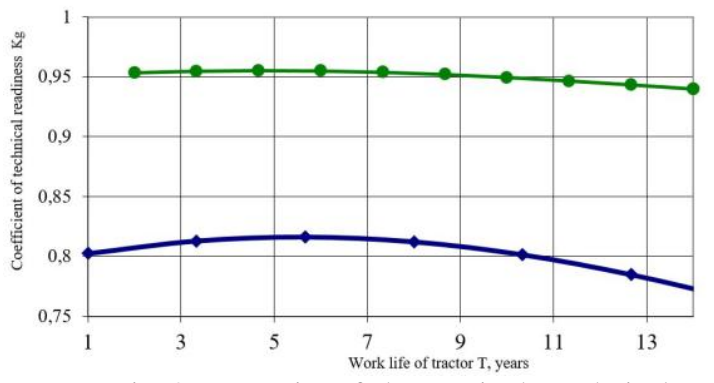

Fig. 3. Dynamics of changes in the technical readiness of tractors (based on the results of neuralnetwork simulation)

From the graph (Fig. 3) it can be seen that the value of the coefficient of technical readiness of the studied tractors is gradually increasing and reaches a maximum by about 5-6 years of service, and after that it is steadily falling. For tractors of the T-402 brand (green line), it is the largest, compared to DT-75 (blue line) they are less idle due to technical malfunctions. Note that under ordinary operation, the coefficient value does not reach unity. For T402 tractors, the maximum value reaches 0.9 , for DT-75 - 0.86 .

\section{DISCUSSION}

Optimization of the MTA use plan is by far one of the most relevant and effective reserves for increasing the economic efficiency of agricultural production, and without capital investments. The mathematical solution to this problem is often reduced to the traveling salesman problem [4, 5] elements of the theory of traffic flows are used [7]. To take into account in the models the difficulties associated with the need for additional time to transfer the MTA from one field to another and reaggregation, it is proposed to introduce additional blocks of separate temporal and spatial planning [9]. The way to mathematically take into account the situation when the base of the territorial location of the equipment may not be the only one was proposed in [10]. Taking into account the particularities of the statement of the resource allocation problem, it is proposed to use different methods of linear programming, in particular, the "simplex method" [13, 14], as well as nonlinear, dynamic, and integer programming. The latter method is most preferable, since the technique is integral units of the resource and their values cannot be fractional.

Despite the variety of models for the optimal distribution of MTA by type of work, they do not take into account the "age" of the equipment, its 
technical readiness, the experience of the tractor driver, and often the current working conditions [18]. This is unacceptable for planning in real production. If all these factors are taken into account, then it is not necessary to distribute the makes of tractors with an indication of their number, but each tractor individually. In this case, the available models of optimal planning for the use of MTA, as well as the mathematical apparatus, are not applicable in practice.

Given this, the authors developed a fundamentally new mathematical model for optimizing the use of technology, which, unlike existing ones, allows to:

- plan the operation of each tractor individually;

- designate the so-called "mandatory tractors", that is, those that the user, at his discretion, would like to assign to a particular operation or vice versa, would like the tractor operator not to work on this operation. This is especially important, since the final decision on the use of technology is made subjectively. The developed model allows subjective amendments to be laid in advance before solving the problem and to obtain the optimal plan being implemented.

To solve the problem, in addition to the model, the authors developed a mathematical apparatus for integer programming with Boolean variables based on the construction of the LS. This method differs fundamentally from the existing ones in the ability to solve the problem without rounding the desired values. To construct the LS, the authors derived a formula for calculating the value of any element.

Based on the results of experimental studies, functional dependencies for adjusting the performance of MTA with the following were identified:

- "age" of the tractor based on the dynamics of its effective power;

- coefficient of technical readiness of the tractor;

- work experience of a tractor driver.

It is established that the power of the engines of tractors of the DT-75 type in the pre-repair period (4000 ... 5000 moto-hours) decreases by $8 \ldots 10 \%$. After a major overhaul, the engine power is not fully restored and is $3 \ldots 4 \%$ lower than the nominal value. The rate of power drop after overhaul is 1.54 times higher than before repair.

The engine power of the T-402 tractors decreases faster and in the pre-repair period (3500 .. 4100 moto-hours) decreases by $13 \%$. After repair, power decreases 1.41 times faster than before repair.

By restoring operational adjustments, it is possible to increase the power of the engines of DT-75 tractors by an average of $2 \%$, and T-402 tractors by $3 \%$. Moreover, after adjustments, the value of the coefficient of determination increases. This confirms the widespread prevalence of unauthorized regulation by the tractor drivers of the engine power system. In particular, we have recorded numerous cases of failure of seals of fuel equipment in order to increase the engine speed.

It is proved that the performance of MTA has a stronger correlation with the experience of the machine operator than with its class and age. To take into account the influence of this factor on aggregate productivity, a relationship between them is formalized, which has an extremum. The maximum performance of the unit is achieved with the experience of the machine operator for 16 years.

For the first time, neural-network modeling was used to study the dynamics of the tractor technical readiness coefficient, and the necessary functional dependencies were obtained.

Other researchers did not conduct experiments to obtain practically applicable functional dependencies for adjusting the performance of the MTA, taking into account the "age" of the equipment, its technical readiness and the work experience of the tractor operator. The developed method and model of the optimal distribution of MTA by type of work forms new branches in the tree of their classification.

Thus, the scientific novelty of the work performed are:

- a technique for operational planning of work and increasing the efficiency of using the MTA, taking into account the technical condition of the tractors, the specific conditions for their functioning and the work experience of the machine operator.

- a mathematical model for solving the problem of the optimal distribution of MTA by type of work and the mathematical apparatus for its implementation;

- a technique for adjusting the performance of the MTA, taking into account the experience of the tractor driver, the service life and technical readiness of the tractor.

The developed mathematical model and analytical dependencies for adjusting the performance of MTA were incorporated into the AgroMaster + computer program. The program received a certificate of Rospatent on official registration. It allows in the conditions of a specific agricultural enterprise to quickly plan the operation of equipment. For practical testing, the program was introduced at the "Metevtamak" agricultural enterprise of the Republic of Bashkortostan. The operational distribution of tractors using the program on such simultaneously performed tasks as steam cultivation, continuous pre-sowing cultivation, winter rye sowing with mineral fertilizers, rolling crops, harvesting corn for silage, transporting silage yielded the following results:

- MTA production rates adjusted according to the developed methodology are on average equal to the actual production. They differ by no more than $10 \%$, while this difference between the standard rate and the actual output reaches $85 \%$;

- the use of technically sound standards as a factor in stimulating the labor of machine operators, made it possible to increase the productivity of 
units by at least $7 \%$ and reduce fuel consumption by $10 \%$;

- due to optimal planning, the total duration of operations decreased by 1.7 times compared to their actual duration. At the same time, the cost of additional products received per 1 motorhour amounted to 7.3 dollars.

\section{CONCLUSIONS}

The developed economic and mathematical model, mathematical apparatus, and functional dependencies are implemented in the AgroMaster + computer program. The program allows in real conditions to calculate the actually possible production of units for specific conditions of their functioning, to draw up an implemented optimal plan for the use of MTP.

For the production verification of the research results, the AgroMaster + program was introduced at the "Metevtamak" agricultural enterprise of the Republic of Bashkortostan.

A production audit showed that the production rates corrected by the developed methodology are on average equal to the actual production. They differ by no more than $10 \%$, while this difference between the standard rate and the actual output reaches $85 \%$.

The use of technically sound standards, being an essential factor in stimulating the labor of machine operators, allows to increase the productivity of units by at least $7 \%$ and reduce fuel consumption by $10 \%$.

Due to optimal planning, the total duration of operations is reduced by 1.7 times compared to their actual duration. At the same time, the cost of additional products received per 1 moto-hour is 7.3 dollars.

Thus, the developed methodology for the optimal planning of the use of technology allows, without significant investment, to reduce the time of agricultural operations, reduce fuel consumption, costs in general, and thereby increase production efficiency.

\section{REFERENCES}

1. Ermak S, Pechenkina T. Group pressure. [Internet]. Moscow: Expert online. Special report. 2019; 44(1140), cited 2019 Oct. 28. Available from: https://expert.ru/expert/2019/44/gruppovoe-davlenie.

2. Balas E. Discrete Programming by the filter method. Operations Research 1967; 15(5): 915-957.

3. Zhao WJ. Comparison of technical and economic characteristics of various transportation modes Cooperative Economy and Technology 2017; 3: 3839.

4. Wu Y, Weise $\mathrm{T}$, Chiong R. Local search for the traveling salesman problem: a comparative study. Proceedings of the 14th IEEE Int1 Conference on Cognitive Informatics \& Cognitive Computing. Beijing, China; 2015, 213-220.
5. Lin B, Sun X, Salous S. Solving travelling salesman problem with an improved hybrid genetic algorithm. Journal of Computer and Communications. 2016; 4: 98-106. https://doi.org/10.4236/jcc.2016.415009.

6. Recht P. A dynamic programming approach for the max-min cycle packing problem in even graphs. Open Journal of Discrete Mathematics 2016; 6: 340350. https://doi.org/10.4236/ojdm.2016.64027.

7. Parwanto N, Morohosi H, Oyama T. Applying network flow optimization techniques to improve relief goods transport strategies under emergency situation. American Journal of Operations Research, 2015;5:95-111. https://doi.org/10.4236/ajor.2015.53009.

8. Luo Z. Path selection of multimodal transport based on multi-objective mixed integer, programming. Journal of Transportation Technologies 2019; 9: 462-473. https://doi.org/10.4236/jtts.2019.94029.

9. Wu C, Zhou L, Qiao P, Wang J. Addressing uncertainty in temporal and spatial scheduling for farm machinery operation. Positioning. Scientific research an academic publisher 2016; 7: 32-40. https://doi.org/10.4236/pos.2016.71003.

10. Allate B. Terminal location models for intermodal transport network optimization. Open Journal of Applied Sciences 2019; 9: 307-315. https://doi.org/10.4236/ojapps.2019.95025

11. Shevtsov V, Lavrov A, Izmailov A, Lobachevskii Y. Formation of quantitative and age structure of tractor park in the conditions of limitation of resources of agricultural production. Technical Papers; 2015. https://doi.org/10.4271/2015-26-0147.

12. Jourquin B. Estimating elasticities for freight transport using a network model: an applied methodological framework. Journal of Transportation Technologies 2019; 2 9: $1-13$. https://doi.org/10.4236/jtts.2019.91001.

13. Rüttimann B. Discourse about linear programming and lean manufacturing: two different approaches with a similar. Converging Rational. Journal of Service Science and Management 2015; 8: 85-91. https://doi.org/10.4236/jssm.2015.81010.

14. Tanaka Y. Using the simplex method for a type of allocation problems. American Journal of Computational Mathematics 2019; 9: 25-31. https://doi.org/10.4236/ajcm.2019.92002.

15. Belahcene S, Marthon P, Aidene M. The pivot adaptive method for solving linear programming problems. American Journal of Operations Research 2018;8:92-111. https://doi.org/10.4236/ajor.2018.82008 .

16. Bibi M, Bentobache M. A hybrid direction algorithm for solving linear programs. International Journal of Computer Mathematics 2015; 92:201-216.

17. Wan J, Wei S. Research on route selection of multiobjective multimodal transport based on hybrid algorithm. Journal of Tianjin University (Natural Science and Engineering Technology Edition), 2019; 52: 285-292.

18. Zainagabdinov RR. The problems of economic and mathematical models using in a machinery and tractor fleet work planning proceedings of the 2-nd regional inter-university scientific and practical conference of young researchers and specialists with presentation in foreign language. Ufa, Bashkiriya, Russia; 2001, 25-26. 
19. Zainagabdinov RR. Improving the efficiency of the use of machine and tractor units by improving their distribution by type of work, taking into account current operating conditions. Diss. ... cand. tech. Science.-St. Petersburg, 2004.

20. Plaksin AM. Substantiation of the frequency of control and tolerance for engine power reduction during tractor operation in agriculture. Unpublished PhD theses. Chelyabinsk, Russia; 1975.

Received 2020-02-16

Accepted 2020-05-12

Available online 2020-05-13

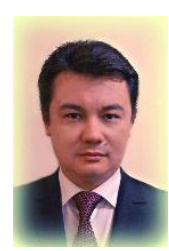

Rishat Rashitovich ZAINAGABDINOV,

Candidate of Technical Sciences, Associate

Professor of the Department of Automobiles and Machine and Tractor Complexes at Bashkir State Agrarian University. Field of research - improving efficiency of the tractors and automobiles

using.

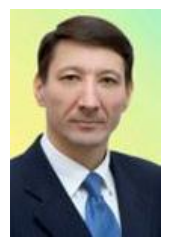

Ildar Ismagilovich GABITOV, Doctor of Technical Sciences, Professor, Honored Scientist of the Republic of Bashkortostan. Field of research: technologies and technical services of automobiles, tractors and mobile agricultural machinery, development of electronically controlled fuel supply system designs, technologies and technical maintenance tools for fuel equipment of automotive diesel engines.

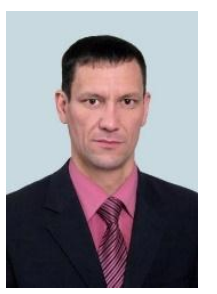

Bakiev Ilshat Talgatovich, Candidate of Technical Sciences, Associate Professor of the Department of Automobiles and Machine and Tractor Complexes at Bashkir State Agrarian University. Field of research improving the maintenance system for tractors and automobiles.

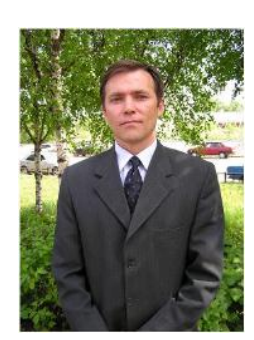

Gafurov Ildar Danilovich, Candidate of Technical Sciences, Associate Professor of the Department of Automobiles and Machine and Tractor Complexes at Bashkir State Agrarian University. Field of research - scientific and informational support of rational use of machinery and oil products in agriculture.

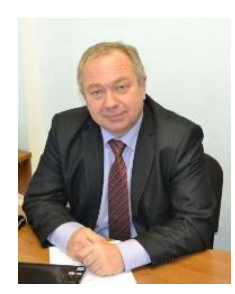

Kostarev Konstantin Vasilievich, Candidate of Technical Sciences, Associate Professor of the Department of Automobiles and Machine and Tractor Complexes at Bashkir State Agrarian University. Field of research - improving efficiency of the tractors and automobiles using. 